\title{
Investigation of a method for real time quantification of gas bubbles in pipelines
}

\author{
K. Baik, ${ }^{\text {a) }}$ T. G. Leighton, ${ }^{\text {b) }}$ and J. Jiang \\ Institute of Sound and Vibration Research, Faculty of Engineering and the Environment, \\ University of Southampton, Southampton, Highfield, Hampshire, SO17 1BJ, United Kingdom
}

(Received 12 August 2013; revised 6 May 2014; accepted 13 May 2014)

\begin{abstract}
The need to measure the dynamic void fraction (the proportion of flowing bubbly liquid that is gas) is common across many power, processing and manufacturing industries. Many such pipelines and liquids are optically opaque, and work on margins that require a low cost solution that is not commensurate with the size of the challenge. Such a solution will therefore be a compromise, and in this paper costs are reduced by using a narrowband acoustic solution that cannot, on its own, contain enough information to characterize the void fraction in real time unambiguously. The ambiguity is reduced using likely estimates of the general shape of the bubble size distribution so that, with a single sourcereceiver pair attached to the outside of the pipe, the absolute gas content can be estimated. While the data that are required a priori (the general shape of the bubble size distribution) are not identical to the output of the inversion (the absolute void fraction of gas entrained as bubbles in the flow), the requirement for such a priori information could limit the usefulness of the technique in industry.
\end{abstract} (C) 2014 Acoustical Society of America. [http://dx.doi.org/10.1121/1.4881922]

PACS number(s): 43.20.Mv, 43.20.Hq, 43.30.Bp [PSW]

Pages: 502-513

\section{INTRODUCTION}

There are many acoustical ways of providing a quantitative estimate of a bubble size distribution (BSD), but all have inherent weaknesses. If these weaknesses can be controlled (e.g., by controlling the bubble size distribution to remove the possibility that large bubbles could be mistaken for smaller resonant bubbles), then the degree to which the measurement conditions violate the assumptions inherent in interpreting the acoustical signals can become insignificant. If, in addition, the user has access to a wide acoustical frequency band, then the weaknesses can often be sufficiently unimportant to allow an adequately accurate estimate of the BSD. Measuring the sound speed, attenuation, or scattering when sound is projected through a bubble cloud can usually provide an estimate of the bubble size distribution, but that estimate can be inaccurate if the range of acoustic frequencies is too narrow, or the bubble size distribution too broad. Ground truth of such estimates is important since convergence of an estimate does not imply accuracy of the estimate. A key question is the extent to which any a priori knowledge of the likely answer, used directly or indirectly to produce convergence in an illconditioned problem, is shared with and compromises the ground-truthing. Such sharing is not always obvious.

Industrial processes that require, or could be improved by, quantification of the bubble population include: filling, pouring, and piping tasks in industry, ${ }^{1}$ the manufacturing and harvesting of products containing bubbles, such as foodstuffs, ${ }^{2}$ cement, ${ }^{3}$ petrochemicals, ${ }^{4}$ pharmaceuticals, ${ }^{5}$ and aerosols; ${ }^{6}$ and the refining and deposition of metals. ${ }^{7}$ Recently, detectors

\footnotetext{
${ }^{a}$ Current address: Centre for Fluid flow and Acoustics, Korea Research Institute of Standards and Science, 267 Gajeong-ro, Yuseong-gu, Daejeon, South Korea, 305-340.

${ }^{b)}$ Author to whom correspondence should be addressed. Electronic mail: tgl@soton.ac.uk
}

for helium bubbles in steel pipelines filled with liquid mercury have been investigated for a spallation neutron source (SNS). ${ }^{8-10}$ The ability to control the bubble size distribution, and to generate and detect a broad range of acoustic frequencies with controlled and calibrated amplitude in order to measure the bubble population, is however more characteristic of the laboratory than of industry.

Bubble populations in pipes can rarely be reliably measured using the currently available acoustic techniques. Most techniques for measuring bubble populations using acoustics assume that the bubbles exist in an infinite volume of liquid, conditions which are never exactly fulfilled. The most popular method (here termed the "free field method") is the comparison of the measured sound speed and/or attenuation with those expected for propagation of plane waves in trains of infinite duration, in an infinite volume of bubble-free water. $^{11,12}$ The free field method works by assuming the discrepancy found in such a comparison is due to the presence of bubbles and inverting that discrepancy to obtain an estimation of the bubble population present. ${ }^{13-15}$ The use of pulses $^{16}$ and the presence of confinement walls ${ }^{17,18}$ mean that in practice these ideal assumptions do not match the measurement conditions. However, if suitably regularized, such inversions can always be made to provide an estimate. This can produce misleading answers. If an independent (e.g., optical) measure of bubble size distribution can be obtained, this can be compared to test the results of acoustic inversion. ${ }^{19}$ However, the independence of the optical data is critical since the regularization of inversions is, in essence, the act of shaping the result based on prior knowledge. If that prior knowledge includes the result against which it is to be tested, the test becomes circular.

Hence, estimations obtained by regularized acoustic inversions when the fundamental physics contains flawed assumptions should be critically assessed for the extent to which (i) the flaws significantly affect the results (sometimes 
they might not) and (ii) the answer is shaped to fit the expected result against which it is subsequently compared. In the case of bubbles in pipelines, the assumption that there exists in bubble-free conditions a single frequencyindependent sound speed and frequency-dependent attenuation (against which to compare the measurements in bubbly conditions) is flawed. This is because the liquid and pipe are acoustically coupled $^{20,21}$ and because, over most of the frequency range of interest in some pipe systems (such as SNS), modal acoustic propagation occurs with distinct frequencydependent sound speeds and attenuations for each mode. The current authors ${ }^{10}$ showed that errors of $+/-1000 \%$ can occur when such an approach is followed in a pipe of similar dimension to that used in the SNS at Oak Ridge National Laboratory (ORNL). Further complications occur with the acoustic inversion techniques that are based on the bubble pulsation resonance since the pipe can alter the inertia, ${ }^{17,22-24}$ frequency, $^{25}$ and damping $^{26}$ of that bubble resonance. In practical pipelines, a wealth of other effects (e.g., sound transmission through the pipe wall, the presence of large gas pockets trapped in pipe junctions; Fig. 1) complicate the issue. ${ }^{27}$

This paper records a test undertaken at a key decision stage half-way into a two-year commission to build acoustic detectors of helium bubbles in the mercury of the SNS Target Test Facility (TTF) at ORNL. At the start of the two-year commission, the budget allowed for a broadband $(1-1000 \mathrm{kHz})$ transducer suite of a type which would wet in
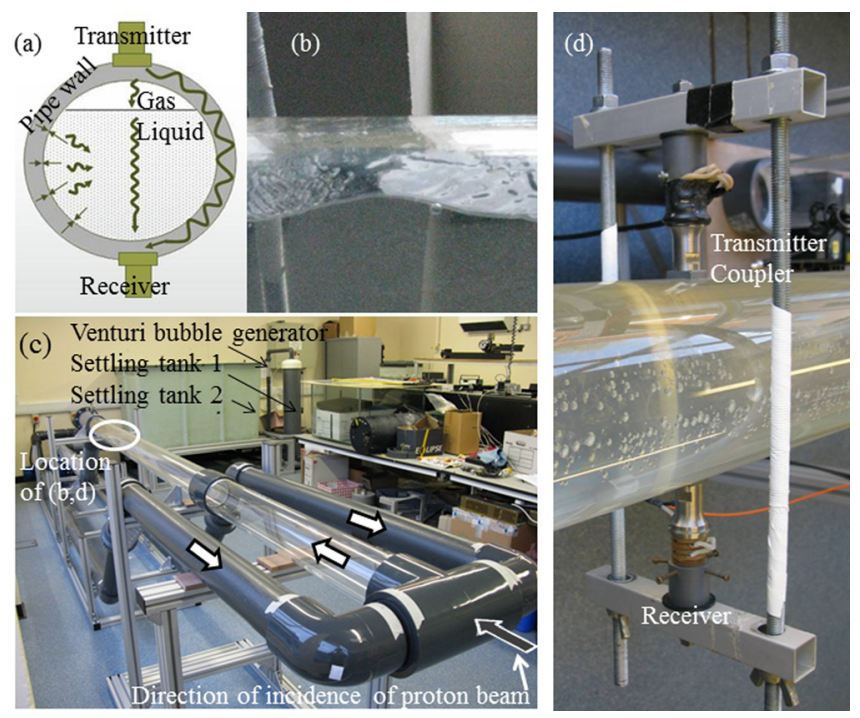

FIG. 1. (Color online) The 1:1 scale experiment (using water in PMMA tubing) of the relevant part of the mercury-in-steel pipework of SNS TTF. (a) Schematic showing the volume of bubbly liquid used in measuring the dynamic void fraction, and the volume (including the gas pocket at the top of the pipe) used by many other methods of measuring "void fraction" (such as from calculations based on the mass of gas injected). Curly arrows show three possible routes for propagation between the transmitter and receiver, both of which are clamped onto the outside of the pipe to allow easy relocation and avoid expensive and potentially hazardous ports into the pipe. (b) A photograph of the gas space which occurred in the region shown by the white ellipse in (c), a full-length photo of the pipe rig in which the liquid flow direction is indicated by white arrows with black borders, and the direction of the incoming proton beam (were this an SNS) is shown by the gray arrow with a white border. (d) Photograph of one of the designs for source and receiver, mounted on a pipe containing flowing bubbly water (the largest bubbles only are visible): one of the couplers which match the flat transducer faceplate to the curvature of the pipe is visible. mercury but not react with it. Acoustic transducers commonly fail to wet properly in mercury, hampering the operation of both transmitters and receivers, and many materials react with mercury in a way that is unacceptable in an environment exposed to hard radiation. The initial intention was to invert the measured sound speeds and attenuation across this frequency range to obtain an estimation of the bubble population. ${ }^{10}$ The first stage of this was to characterize propagation in bubble-free pipelines at SNS TTF, which further showed that useful data could be taken on 1:1 scale model pipelines built of water-filled polymethylmethacrylate (PMMA) pipes (Fig. 1). Here, the coupling between liquid and wall generates similar effects to the coupling seen in SNS TTF. ${ }^{20,21}$ Having therefore (i) characterized the bubble-free baseline ${ }^{20,21}$ and (ii) shown that application of the "free field" inversion method (when bubbles are added) can produce overcounts and undercounts of $+/-1000 \%$ in bubble numbers, ${ }^{10}$ a new inversion was produced that is applicable to data taken in pipes. ${ }^{10}$ The plan to implement this inversion with a broadband acoustic source was, however, interrupted: mid-way through this study the new funding pressure on the sponsors made the planned $1-1000 \mathrm{kHz}$ frequency range unaffordable. The new inversion therefore served two purposes. First, it provided the algorithm by which a suitably funded possible future broadband system, operating over a wide enough frequency range, could estimate the bubble population. ${ }^{10}$ Second, it was used to show that, if the algorithm were to be applied to SNS TTF data over an affordably reduced frequency range, the accuracy would be not acceptable. ${ }^{10}$ This meant that the second year of the commission could not proceed along the broadband inversion route and needed a new direction.

The first major obstacle in devising an alternative bubble detector was to reduce transducer costs by a factor of 100 . First, the intended broadband system was replaced with a single frequency system, swapping the ability to measure the bubble size distribution for the ability to measure the "dynamic void fraction"- the proportion of flowing bubbly liquid that is gas - a substitution that was acceptable to ORNL. Second, costs were reduced by developing a system in which the transducers could be clamped onto the outside of the steel pipe, rather than be immersed in the mercury. This removed the need (i) to build transducers out of materials which would be chemically stable but completely wetted in contact with mercury and (ii) to drill ports in the ORNL pipeline, which is costly to do safely for a mercury-filled facility. This provided the added bonus that the sensors were not fixed in place, but could be relocated to any point on the pipeline in minutes in order to locate and address problem areas.

Bubbles injected into mercury are subject to strong buoyancy forces and would have a tendency to rise out of the flow, coalesce and form large gas pockets. While the opacity of steel and mercury make it difficult to check for gas pockets throughout the ORNL pipeline, it was observed in the 1:1 scale model of the ORNL pipeline built at the University of Southampton using PMMA pipes and water [Fig. 1(b)]. At the time of testing, a window was placed in the target upper wall of the SNS TTF. However, bubbles rising up against a window so as to be visible could imply the presence of a "dynamic void fraction" in the liquid (in that 
bubbles are present in the flowing liquid and some rise up to the window) or its absence (all bubbles are too large to stay entrained in the flow and all are rising up to the window).

Safety considerations meant that the scale model test was undertaken on water-filled PMMA pipes at the University of Southampton, rather than the mercury-filled steel pipelines of ORNL: earlier studies ${ }^{10,20,21}$ had shown that while the coupling between pipe wall and content was not identical in the two cases, it was sufficiently similar to test principles in this way.

The objectives were (i) to find a signal that primarily reflected only the dynamic void fraction, immune from the presence of any large gas pockets, and that was robust with respect to noise, (ii) to do so in real time using a pair of single-frequency off-the-shelf transducers clamped onto the outside of the pipeline, for a fraction of the original budget, and (iii) assess to what extent this signal can be inverted to provide an absolute measure of the dynamic void fraction.

\section{EXPERIMENT}

It is the richness of waves present, when sound propagates in a fluid that is well-coupled to the pipe wall, which makes traditional inversion methods become so inaccurate if they are not adapted to account for the loss of free field conditions. ${ }^{10}$ To build an inexpensive but accurate alternative that operates at only a single frequency, the method devised was one that, rather than ignore that richness as the traditional methods would, instead exploits it to obtain the dynamic void fraction from measurements at a single frequency.

This paper describes a so-called "Chi-beam sensor": to distinguish it from other sensor configurations, the "Chi" label was used by the authors as short-hand for "cross-section," in recognition that here an appropriate cross-sectional schematic of the pipe can include both transducers (source and receiver) and the axes of their main beams, which are perpendicular to the pipe axis. The transducers are mounted across the pipe with an angle $\varphi$, which was $180^{\circ}$ in this experiment but kept as a variable for the theoretical analysis. Energy will travel from source to receiver by numerous paths, through the pipe wall, the liquid contents, and the interface between the wall and liquid. A wide variety of compression, shear and surface waves can contribute to the signal detected by the receiver [Fig. 1(a)]. Separating out the energy contributions at the receiver from each wave would be a mammoth task, and attempts to do so by propagating pulses and allowing the different wavespeeds to sort out these contributions in the time history would be futile because the propagation paths are so short and the reverberation so long.

The solution is to embrace all of these waves and, furthermore, excite the pipe wall continuously rather than in pulses. The energy that the source imparts to the system is transferred to the receiver, which is analyzed using a partial wave series. The tube system can be stiffer or softer depending upon the frequency and the material parameters of the tube and the potentially multiphase liquid flowing inside the pipe. Consequently the acoustic system, defined as the regions of the pipe and its contents through which significant sound transmission occurs between source and receiver, changes as the population of bubbles within the flowing liquid changes. This in turn modifies the acoustic properties of the liquid inside the tube and subsequently changes the stiffness of the pipe system containing the bubbly liquid. Even small dynamic void fractions of bubbles (e.g., $<10^{-5}$ ) inside the liquid will significantly change the bulk modulus of the liquid at the frequencies of interest here. For example, in water that contains a monodisperse population of 0.14-mm-radius air bubbles, the bulk modulus of the bubbly water can differ from that of bubble-free water by a factor of $\sim 3.5$ for $24 \mathrm{kHz}$ sound waves when the void fraction is only $10^{-5}$. Such changes in the acoustic system (the propagation paths and boundary conditions) are reflected in the pressure detected by the receiver. Treatment of the complete system (the pipe, its contents, and the two transducers) as a single complex dynamic entity in this way obviates the need to know and model in detail all the many propagation paths between source and receiver, including compressional waves in the pipe and its contents, interface waves, and distortions of the pipe cross-section.

The efficacy of this approach can be seen in the following manner. If no bubbles are in the flow, or if the only gas is contained in a large trapped gas pocket at a junction or against the top wall of the pipe, then the statistics of the signal detected by the receiver do not change [Fig. 2(a)]. The bubble detection algorithm must interpret that lack of change as a lack of small bubbles in flow above its void-fraction detection threshold (colloquially, the "no-bubble" case). If, however, bubbles pass through the pipe, the stiffness of the system changes in time, and the received signal is perturbed [Figs. 2(b) and 2(c)]: the algorithm must quantify the perturbation in terms of the dynamic void fraction. There is no need to compare the signal with bubbles present against the signal in bubble-free conditions as there is with traditional methods, and measurements need only be taken at single frequency, as opposed to a the two decades or more usually used. ${ }^{13,14}$

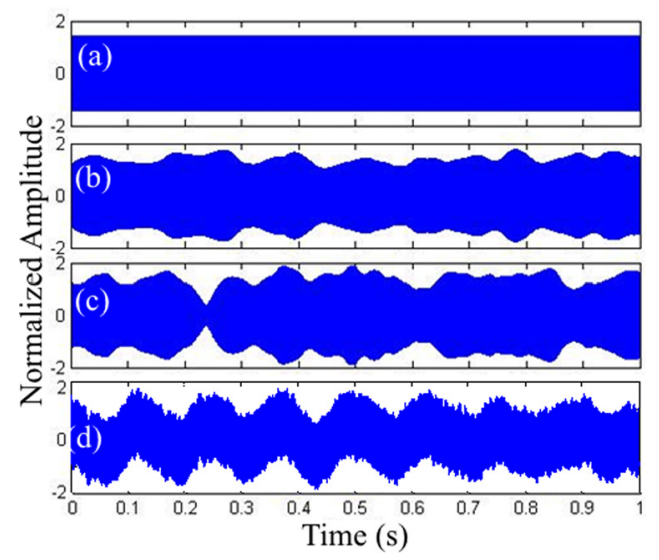

FIG. 2. (Color online) Experimental data from the water-filled PMMA pipe showing the effect on the received signal when bubbles pass through the pipe, the receiver and transmitter both being clamped to the outside of the pipe and connected to it by the couplers. The bubbles modulate the envelope of the continuous monochromatic carrier wave that the transmitter produces. The graphs show the normalized Chi-beam output signals with a fluid/gas mixture in the following cases: (a) bubble free, (b) with a dynamic void fraction $\left(\Gamma^{\prime}\right)$ of $1.3 \times 10^{-4}$, (c) $\Gamma^{\prime}=4.2 \times 10^{-4}$, and (d) bubble-free with added noise. The timescale of the plot is such that the $41 \mathrm{kHz}$ carrier wave plots as a solid band and only the amplitude modulation of this carrier wave is visible. 
Furthermore, the sensor monitors the dynamic void fraction continuously and can respond on subsecond timescales to changes in the bubble population; this advantage would be key to the studies of the type planned at ORNL. There, different gas injectors and gas injection time series would be compared. A sub-second response time in the detector would allow the effect on the dynamic void fraction of changes to the bubble injection conditions (nozzles, gas pressure, etc.) to be monitored in real time, allowing testing and diagnosis of such bubble injection systems. Broadband acoustic techniques, and optical techniques, require a time window (from seconds to minutes) to sample the field in order to obtain an estimate of the bubble size distribution, and the results often require off-line post-processing to produce.

The 1:1 scale water-filled pipe apparatus was used to test the immunity of the sensors to noise and flow, the presence of gas pockets trapped at junctions, and relative changes of the output with pump speed. Noise (whether electrical or vibro-acoustic) breaks the symmetry of the envelope [Fig. 2(d)] and so is easily distinguished, even in high noise environments, from the bubble-induced perturbations that have reflective symmetry about the baseline [Figs. 2(b) and 2(c)]. Calculation of the dynamic void fraction by demodulation extracts just this symmetry, so that noise [Fig. 2(d)] is not included in the calculation since contributions by noise lack such reflective symmetry, and so demodulate to zero.

It was not possible to deploy a non-acoustic measure of void fraction in the 1:1 scale water-filled pipe apparatus that was sufficiently accurate over two orders of magnitude in void fraction in order to test independently whether the dynamic void fraction inferred from the Chi-beam signal was an accurate measure of the actual void fraction in the pipe. Therefore, to implement a non-acoustic void fraction measurement, experiments were carried out in a vertical PMMA tube with the experimental layout of Yim and Leighton. ${ }^{1}$ In this experiment, the tube is $2 \mathrm{~m}$ in length, $4.445 \mathrm{~cm}$ in the inner radius and $5 \mathrm{~cm}$ in the outer radius (Fig. 3). Such tubes have previously been shown to exhibit the important features (subsonic and supersonic modes, break points, etc.) that can be expected of the SNS TTF mercury-filled steel pipeline. ${ }^{10,20,21}$ The tube is sealed on one end and is filled with water. Air bubbles are generated via a needle mounted $\sim 20 \mathrm{~cm}$ from the bottom, and the total void fraction of bubbles is altered by varying the air flow to the needle form an aquarium air pump (Rena Air-200). The BSD could be measured by the optical method described (with results) in Ref. 10, and gave a range of bubble sizes typically from $30 \mu \mathrm{m}$ to $1 \mathrm{~mm}$ distributed in this pipe system. This realistically matches the difficulties of the ORNL problem, whereby for monochromatic insonification, some bubbles will be driven at, some above, and some below resonance. For any given void fraction, the relative numbers of small and large bubbles can be altered by use of a mobile phone vibrator placed on the needle. ${ }^{28,29}$ The sensor pair is mounted face to face across the tube about $1.5 \mathrm{~m}$ from the bottom. The transmitter is driven by a signal generator and the signal collected by a receiver is recorded by a DAQ system. Meanwhile, bubbles are collected at the top of the tube by a funnel to provide a direct and independent measurement of the void fraction.

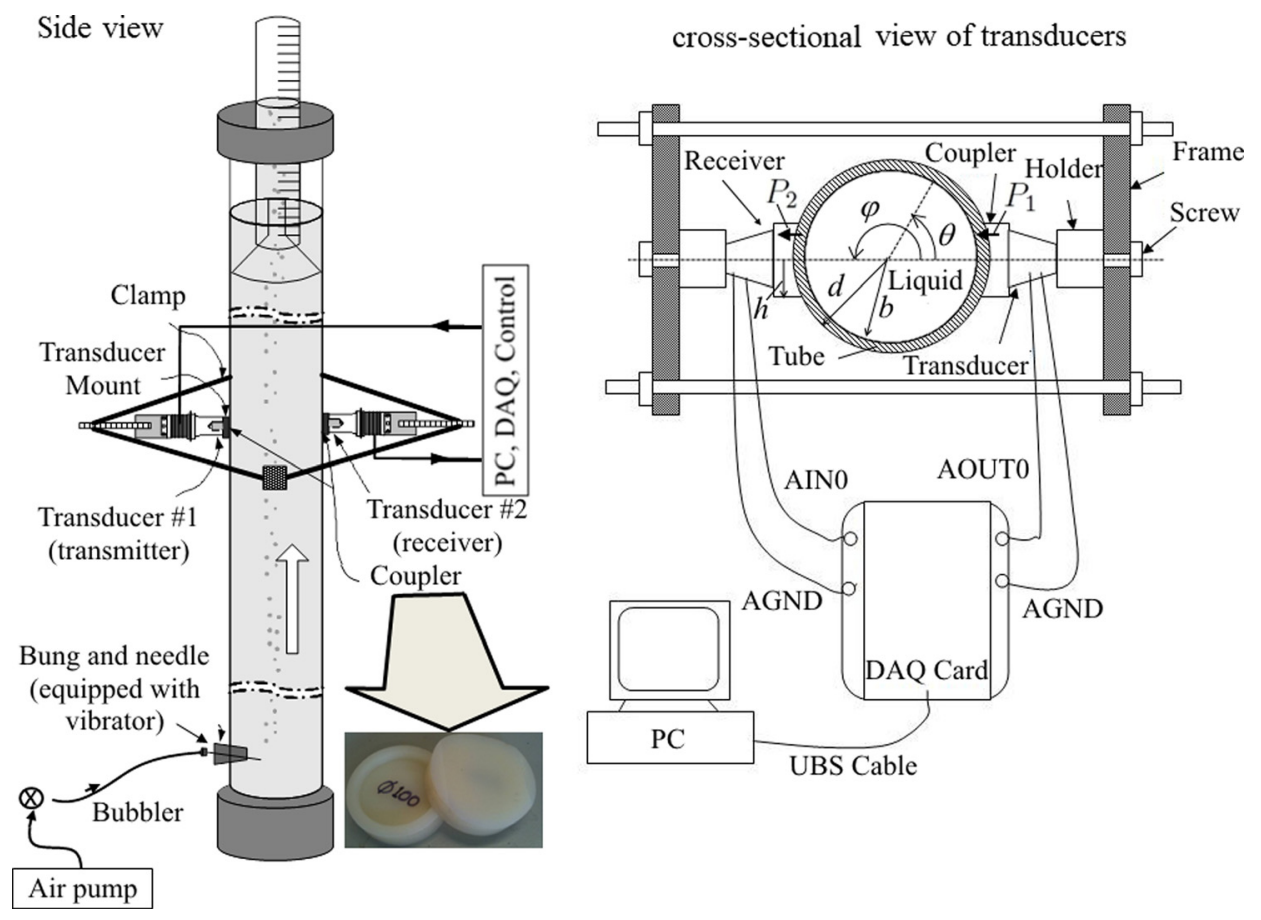

FIG. 3. (Color online) Side view of the vertical pipe test apparatus (with cross-sectional view at the level of the transducers) used for validation of the sensor (not to scale). In the cross-sectional view, the $z$ axis is perpendicular to the page (out of page). The inner and outer radii of the pipe are $b$ and $d$, the transducers are mounted in-plane with the pipe cross section and the angle between their beams is $\varphi$, and $\theta$ is the azimuthal angle relative to the source axis. The radius of each coupler is $h$. A pressure, $P_{2}$, is measured by the receiver in response to a pressure $P_{1}$ applied by the source. The coupling devices which match the shape (and to a lesser extent, the impedance) of the transducers to the pipe wall, are detailed in the photographic inset. The coupler on the left reveals the flat boundary to the transducer and the coupler on the right shows the curved boundary that matches the pipe wall. ORNL hoped to introduce acoustic absorption through bubble injection, and this apparatus was adapted to demonstrate this in a student teaching demonstration (Refs. 28 and 29). 
In this experiment, the Chi-beam sensor pair is driven at a frequency of $41 \mathrm{kHz}$ (the resonance of the high- $Q$ transducers that were adventitiously available at low cost). To be detected appropriately by the Chi-beam sensor, the bubbles should reside sufficiently long within the region of the pipe where the cross-section modes induced by the sensors are formed. In our system this region is typically characterized by the size of the coupler (here, $5.5 \mathrm{~cm}$ diameter). Even a bubble as large as $1 \mathrm{~mm}$ radius, which is rare in our system, will take $180-360 \mathrm{~ms}$ to cross this region. ${ }^{30}$ Almost all the bubbles in this study are smaller, and will stay within the detection range longer than $\sim 200 \mathrm{~ms}$ that is the energy storage time of our narrow sensor [as estimated from the $Q$-factor of the sensor ( 8500 as measured in situ) and the driving frequency of our sensor $(41 \mathrm{kHz})]$. This means that the energy change due to the pulsation of the bubbles is effectively detected by our narrow band sensor system.

To reduce the noise from the pump, background and electronics, a band pass filter is applied to isolate the signal at the drive frequency only, because the modulation caused by bubbles is not influenced by noise from other frequencies. Recall, however, that the basic physics of the signal meant that noise, lacking reflecting symmetry about the baseline, is not in principle included in the calculation of dynamic void fraction (Fig. 2). Modulation of the envelope, with reflective symmetry about the baseline, in ORNL TTF should come only from the presence of bubbles in the mercury passing through the cross-sectional area where the Chi-beam detector is installed, as a consequence of bubble-induced changes in the elastic properties of the pipe-liquid system.

To quantify the component of the fluctuation which has reflective symmetry and therefore is due to bubbles, a normalized envelope integration value (EIV) is used here and can be expressed as EIV $=(1 / T) \int_{T}\left\|H\left(p^{\prime}\right)\right\| d t$, where $H$ is Hilbert transform and $p^{\prime}$ is the normalized Chi-beam output signal, which does not have a DC offset.

In order to measure the void fraction inside the pipe, a funnel was installed on the top of the tube as shown in Fig. 3. The funnel begins to collect bubbles when the pump switched off until the moment that all the air inside the tube is eventually collected. The estimate of the dynamic void fraction obtained from this funnel measurements $\left(\Gamma_{\text {funnel }}\right)$ can be calculated by $\Gamma_{\text {funnel }}=V_{\text {air }} /\left(V_{\text {air }}+V_{\text {water }}\right)$, where $V_{\text {air }}$ is volume of collected air after the pump switched off and $V_{\text {water }}$ is volume of water inside the tube after all the air was collected by the funnel at the top.

\section{THEORY}

The objective is to estimate the dynamic void fraction from the EIV derived from the Chi-beam sensor. To investigate the relationship between the signal sent by the source and the one received by the receiver, the circumferential modes (when $k_{z} \rightarrow 0$, where $k_{z}$ is the axial wavenumber along the $z$ direction) of the liquid-filled tube are examined using a partial wave series. It is assumed that the source excites the tube, and the receiver records the response, with only planar motion across the faceplate of each transducer. The route to adapt the theory for phase changes across the transducer is clear but its execution would be laborious, and in this case unnecessary. By considering the pipe wall as a structure coupling to the sensor pair as well as the liquid inside, the output of the sensor (as detected at the receiver location) responds to the acoustical boundary condition at the sensor position and loading on the inner wall. When only planar excitation is considered, the pressure at the source location, $P_{1}$, can be expressed by partial wave series as

$$
P_{1}=\sum_{n=0}^{\infty} p_{1 n} \cos n \theta e^{-i \omega t}, \quad p_{1 n}=\frac{\varepsilon_{n}}{\pi} \int_{0}^{\pi} P_{1}\left(\theta^{\prime}\right) \cos n \theta^{\prime} d \theta^{\prime},
$$

where $\theta$ is the azimuthal angle relative to the source axis (Fig. 3 ), and where $\varepsilon_{n}$ equals 1 at $n=0$ and 2 otherwise (where $n$ is a non-negative integer). For a point source, $P_{1}\left(\theta^{\prime}\right)=P_{10} \delta(\theta)$ and $p_{1 n}=P_{10} \varepsilon_{n}$. The force caused by the transducer, $F$, is the product of the radiation impedance, $Z_{r}$, and the particle speed of the adjacent media, $v$, as ${ }^{31} F=Z_{r} v$. Consequently, the pressure at the receiver, $P_{2}$, can be expressed as $P_{2}=i \omega z_{2 a} u_{r}^{e}(\theta=\varphi)$, where $z_{2 a}$ is the specific acoustic impedance of the receiver and $u_{r}^{e}(\theta=\varphi)$ is the radial component of the displacement vector of the elastic solid (tube) at $r=d$ caused by the excitation of $P_{1}$. This equation shows that, for a given acoustic excitation, the measured pressure is dependent on the acoustic impedance of the receiver and the magnitude of the normal displacement of the pipe at the location where the receiver is mounted. Once the driving frequency is fixed so that the acoustic impedance of the receiver is fixed, the measured pressure is purely dependent on the magnitude of the normal displacement which is determined by the following matrix equation: ${ }^{32}$

$$
\begin{aligned}
u_{r}^{e}(\theta)= & \frac{1}{2 \mu_{e}} \sum_{n=0}^{\infty} \frac{p_{1 n}}{D_{n}}\left[\left(-k_{c} J_{n+1}^{c d}+\frac{n}{d} J_{n}^{c d}\right) D_{n}^{(1)}\right. \\
& +\left(-k_{c} Y_{n+1}^{c d}+\frac{n}{d} Y_{n}^{c d}\right) D_{n}^{(2)}+\left(\frac{n}{d} J_{n}^{s d}\right) D_{n}^{(3)} \\
& \left.+\left(\frac{n}{d} Y_{n}^{s d}\right) D_{n}^{(4)}\right] \cos n \theta,
\end{aligned}
$$

where $\mu_{e}$ is shear modulus of the pipe wall, and $k_{c}$ and $k_{s}$ are the longitudinal and shear wavenumbers in the tube material, respectively. The Bessel functions, $J_{n+1}^{c d}$ and $Y_{n}^{\text {sd }}$, are simplified notations for $J_{n+1}\left(k_{c} d\right)$ and $Y_{n}\left(k_{\mathrm{s}} d\right)$, respectively. The elements of the matrix, $D$, that originate from the elastic boundary conditions on the tube's inner surface (where there is acoustic coupling between the pipe and the liquid) and its outer surface (involving the pipe only), ${ }^{29}$ are obtained from Eqs. (7a)-(7g) in Ref. 32 and its determinant is denoted as $D_{n}$. The acoustic properties of the liquid are associated with the elements of $4 \times 4$ matrix, $D$, that are the non-zero elements in Eqs. (7a)-(7f) of Ref. 32 when $k_{z} \rightarrow 0$. Thus, only $d_{i j}$ in Eqs. (7a)-(7f) of Ref. 32 where $i, j=1,2,5,6$ are considered as a matrix element of $D$ with the condition of $k_{z} \rightarrow 0$. The determinant, $D_{n}^{(m)}$, is obtained by substituting the $m$ th column of matrix $D$ with the transposition of the vector, $\left[\begin{array}{llll}0 & 0 & 0 & 1\end{array}\right]$. The introduction of bubbles into the liquid changes $Q_{n}$ in Eq. (7e) of Ref. 32 with the condition of $k_{z} \rightarrow 0$ 
which substitutes wavenumber $k_{0}$ in pure liquids with $k_{m}$ in bubbly liquids. Explicit form of the matrix elements can be found from Ref. 33.

Although the evaluation of Eq. (2) is ideally an infinite summation, each actual summation is truncated up to the maximum of the mode index, $n$, which satisfies $n \leq \pi /$ $\left[2 \sin ^{-1}(h / d)\right]$. Here, $h$ is the half width of the flat side of the coupler, which is a machined plastic coupler that is flat at its boundary to the transducer but curved to match the boundary with the pipe (Fig. 3). This relationship follows from the condition that the size of the aperture should be less than half the wavelength of the circumferential waves along the outer surface of the tube, which allows the mode to be excited by a piston-like transducer.

The issue now is the route by which the wavenumber in bubbly liquid, $k_{m}$, is evaluated in the formulation, as this will determine in large part the a priori information required to cause the algorithm to converge to an acceptable answer. This paper examines the advantages and disadvantages of using the formulation developed by Commander and Prosperetti. ${ }^{23}$ It will now be shown that such an approach requires an estimate of the shape (but not the absolute bubble numbers) of the BSD, in order to invert signals of the form shown in Fig. 2, to estimate the dynamic void fraction.

While physically the equilibrium bubble radius $R_{0}$ is a continuous variable, to make use of a BSD in a numerical calculation of this sort it must be discretized. The discretization is performed by representing the BSD $n_{b}\left(R_{0}\right)$ by the histogram $n_{b}\left(R_{0 m}\right)$, where $R_{0 m}$ is the bubble radius at the center of the $m$ th radius bin, the bubble size which is used as proxy for all the bubbles that fall into the domain of the bin under consideration. Each $\operatorname{bin}^{14}$ has width $\Delta R_{0}$. In such a discretized scheme the dynamic void fraction, $\Gamma^{\prime}$, is $\Gamma^{\prime}$ $=4 \pi \Sigma_{m} R_{0 m}^{3} n_{b}\left(R_{0 m}\right) \Delta R_{0} / 3$.

Practical implementation of the scheme requires some compromises, understandable since the goal was to produce a useful sensor for one fifth of the original budget. The savings made by reducing the bandwidth from three decades in frequency to a monochromatic excitation means that such compromises are unavoidable. Unique interpretation of the Chibeam signal in terms of the bubble population is not possible: the received modulated single carrier frequency does not have
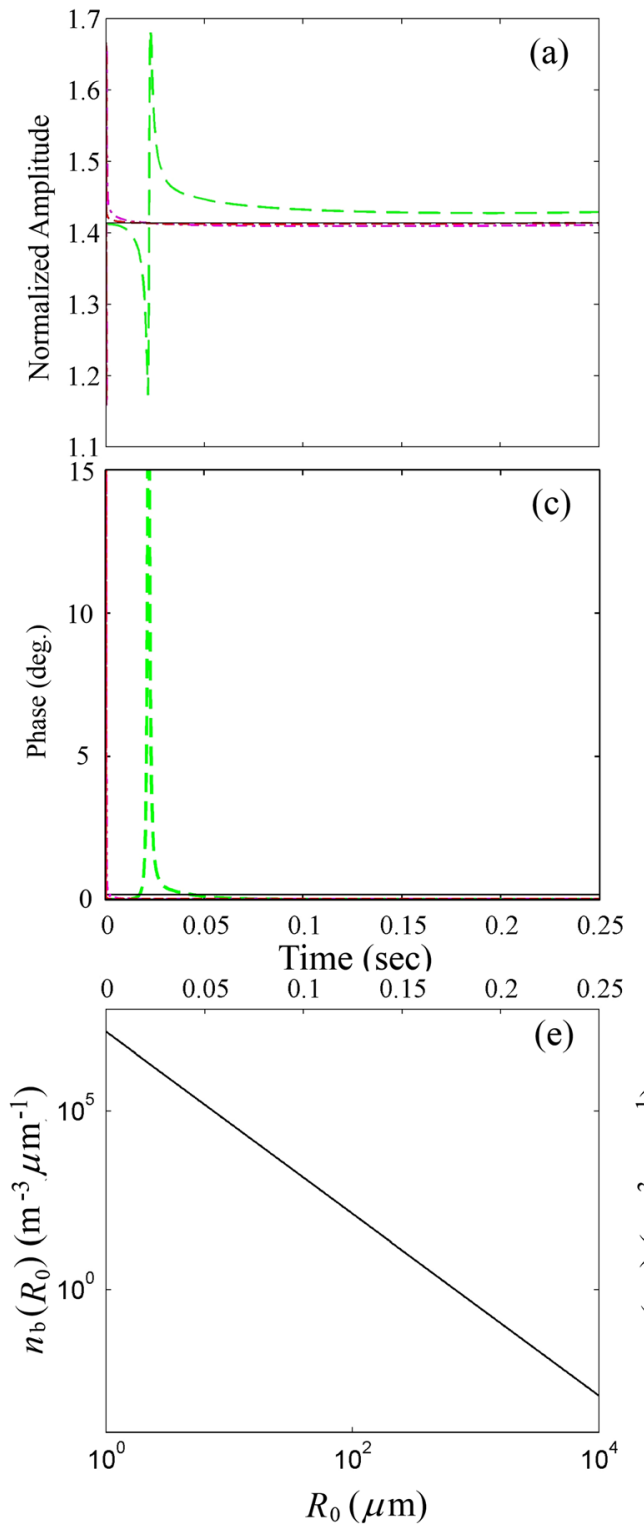
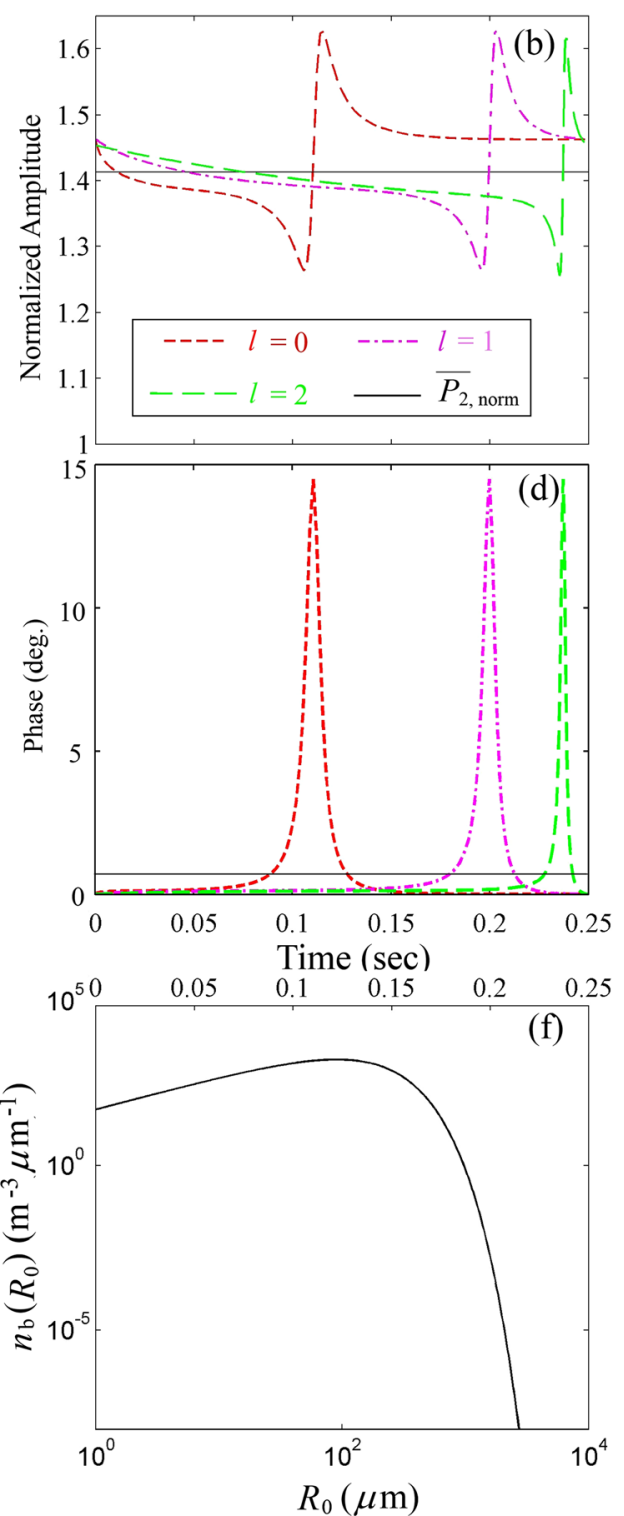

FIG. 4. (Color online) Panels (a) and (b) show examples of the normalized amplitude $\left(P_{2, \text { norm }}\right.$, broken lines), and the average $\left(\bar{P}_{2, \text { norm }}\right.$, solid lines) of the normalized amplitude, in the simulated time history in the PMMA/water tube, for a $41 \mathrm{kHz}$ carrier wave when the shapes of $n_{\mathrm{b}}\left(R_{0}\right)$ in (e) and (f), respectively, are used with an input dynamic void fraction of $3 \times 10^{-5}$. Under the same conditions and parameters used in panels (a) and (b), the phases of the simulated time histories are displayed, respectively, in panels (c) and (d). (e) shows a BSD of the form $n_{b}\left(R_{0}\right)$ $\propto R_{0}^{-\alpha}$, where $\alpha=2.55$ and $R_{0}$ is sampled from $1 \mu \mathrm{m}$ to $1 \mathrm{~cm}$. (f) plots a BSD that is a Boltzmann distribution with a peak bubble radius of $R_{p}=90 \mu \mathrm{m}$, with the shape of the BSD is described by $n_{b}\left(R_{0}\right) \propto R_{0} \exp \left(-R_{0} /\right.$ $\left.R_{p}\right)$. Three different values of $l$ are used to test the effect of deviations from a quadratic dependence $[l=2]$ of the received signal on the bubble radius (which would physically occur if the contributions were to add incoherently, and the contribution from a single bubble were to depend on the cross-sectional area $\left[\pi R_{0 m}^{2}\right]$ or bubble wall area $\left.\left[4 \pi R_{0 m}^{2}\right]\right)$. Assuming, therefore, that the received signal is proportional to $R_{0 m}^{l} n_{b}\left(R_{0 m}\right)$, the use of small dashes indicates an assumption of $l=0$; use of dash-dot lines indicates $l=1$; and large dashes indicate $l=2$. The EIV is calculated by the area enclosed by $P_{2 \text {,norm }}$ and $\bar{P}_{2 \text {,norm }}$, which gives (a) $0.0020(l=0), 0.0129(l=1)$, and $0.0108 \quad(l=2)$ and (b) 0.0419 $(l=0), \quad 0.0283 \quad(l=1), \quad$ and 0.0233 $(l=2)$. 
the bandwidth to accommodate the ambiguity arising from the fact that a resonant bubble may be mistaken for a large nonresonant bubble (and vice versa) since they both scatter strongly. This problem could in principle be solved by ensuring that no bubbles are resonant through use of a frequency that is much higher than, or much lower than, that of any bubble resonance. The option of using very high carrier frequencies is not available for SNS TTF because (i) very high frequencies would be attenuated too strongly for sources and receivers clamped to the outside of the pipe and (ii) without knowing the maximum bubble size present in what is likely to be a wide distribution of bubble radii, high frequencies might cause violation of the assumption that the bubble radius is always much smaller than the acoustic wavelength, an assumption that underpins much of bubble acoustics. ${ }^{34}$ The inability to guarantee that there are no bubbles resonant at the carrier frequency is partially mitigated by driving the monochromatic source at a number of frequencies (harmonics of the transmitter's fundamental, available at no extra cost) to check that they all give the same void fraction estimation. If a sufficient number of bubbles were to be resonant at one of the harmonics, the inversion would give different void fractions at different frequencies.

The compromises are implemented by including the following important assumptions in the Chi-beam algorithm presented in this paper. First, any nonlinear contributions by bubbles are disregarded so that the measurements can be described by the summation of linear contributions from each bubble. Second, the time-averaged Chi-beam signal level represents the average contribution by each size of bubble within the pipe. Finally, assume bubbles are moving during the counting process. The buoyant rise velocity of a bubble, which depends on its bubble radius, must be vectorially added to the time-average bulk flow velocity of the liquid. The bulk flow velocity of the liquid will be aligned with the bubble rise velocity in vertical pipes but not in horizontal pipes. Consequently, because the sensor performs a local (as opposed to a global) count along the pipeline, here we assume that the counting rate is proportional to the $l$ th power of bubble radius, where the appropriate value of $l$ will be explored in this paper. In statistical terms, the contribution to the received signal by bubbles with the size of $R_{0 m}$ which will be designated $P_{2, m}=P_{2}\left(R_{0 m}\right)$, is proportional to $G_{m}=R_{0 m}^{l} n_{b}\left(R_{0 m}\right)$. If the contribution of a single bubble to the received signal depended on the $2 \mathrm{D}$ cross-sectional area of the bubble $\left(\pi R_{0 m}^{2}\right)$ or the wall area of the bubble wall $\left(4 \pi R_{0 m}^{2}\right)$, then $l=2$ would be appropriate if their contributions add incoherently. However the details of bubble scatter ${ }^{34,35}$ suggest that rather than assume such a relationship a priori, it would prudent first to explore the possibility of deviations from this quadratic relationship.

The instantaneous signal received by the sensor, $P_{2}$, is obtained by substituting $k_{1}$ with $k_{1}^{\prime}$ in Eq. (2.1) of Ref. 10, which is the same as the formulation by Commander and Prosperetti. In the current paper, note that $k_{1}$ and $k_{1}^{\prime}$ are represented by $k_{0}$ and $k_{m}$, respectively. Consequently, the deviation of the Chi-beam signal caused by bubbles in a particular size bin from bubble-free conditions is proportional to $\left|P_{2, m}-P_{2, \text { nobub }}\right| G_{m}$. Here $P_{2, m}$ is the amplitude of the signal measured by Chi-beam sensor if only bubbles with size of $R_{0 m}$ were to exist within the pipe and $P_{2 \text {,nobub }}$ is the signal level detected by the Chi-beam sensor in the bubble-free condition.

Therefore the time-averaged Chi-beam measure of the amplitude fluctuation (EIV) between the bubbly and the bubble-free conditions is statistically obtained as

$$
\mathrm{EIV} \approx \sum_{m}\left|P_{2, m}-P_{2, \text { nonbub }}\right| G_{m} / \sum_{m} G_{m}
$$

The amplitude $P_{2, m}$ is theoretically calculated from Eq. (2) if it is assumed that the shape (but not the absolute bubble numbers) of the $\operatorname{BSD} n_{b}\left(R_{0 m}\right)$ has been estimated a priori. Once the shape of the BSD is assumed, simple scaling relates it directly to the dynamic void fraction, $\Gamma^{\prime}$. In this way, the void fraction is related to the amplitude $P_{2}$ of the Chi-beam signal, and by Eq. (3) is further related to the calculated EIV.

Rather than wait for sufficient funds to become available to implement the broadband technique ${ }^{10}$ that would measure the BSD, the pragmatic route is to implement the affordable Chi beam technology despite the clear limitation of requiring an a priori estimate of the shape of the BSD. Such estimates could be obtained using optical techniques (if such technology exists in the industry), with appropriate testing of the robustness of the estimated void fraction to a variety of BSD shapes.

Pragmatism is also required when addressing the theoretical requirement in using Eq. (3) for a measurement in the "bubble free" condition $\left(P_{2, \text { nobub }}\right)$. A large facility like SNS TTF cannot be degassed at will during routine usage. However, when no bubbles are present, the carrier wave should not be perturbed [Fig. 2(a)] except by noise, and so, in the absence of bubble-free data, an estimate of the bubblefree condition can be obtained by averaging the received signal. Basing the detection on envelope perturbation, rather than the absolute signal, also reduces the need to characterize in full the impedance mismatches and losses associated with transducer, coupler, the pipe and its contents, and allows the system to be moved from one pipe type to another without recalibration. To implement these protocols and so avoid the practical difficulties, instead of using Eq. (3) which requires $P_{2, m}$ and $P_{2, \text { nobub }}$, in the current study we used the normalized amplitude $P_{2, \text { norm }}$ and its average $\bar{P}_{2 \text {,norm }}$ to calculate the amplitude fluctuation. The normalized amplitude $P_{2, \text { norm }}$ is calculated by normalizing $P_{2, m}$ with respect to its standard deviation. The standard deviation of the $P_{2, m}$ over one time period is designated STD and statistically given as

$$
\mathrm{STD} \approx \sqrt{\sum_{m} P_{2, m}^{2} G_{m} / 2 \sum_{m} G_{m}} .
$$

If $P_{2, m}$ is constant, STD is reduced to $1 / \sqrt{2}$ and normalizing by this factor gives the amplified signal level divided by a factor of $\sqrt{2}$. Therefore, the normalized signal level is $P_{2, \text { norm }}=P_{2, m} /$ STD. This eliminates the dependence of the signal level on the acoustic impedance of the receiver and the normalized signal level is purely determined by the normal displacement at the receiver location. Therefore, the normalized EIV is the time average of the absolute value of the 
deviation of $P_{2 \text {,norm }}$ with respect to $\bar{P}_{2, \text { norm }}$. With these practical considerations, the formulation to calculate the normalized EIV which relates the response of the Chi-beam signal to the dynamic void fraction is given by

$$
\mathrm{EIV}_{\text {norm }} \approx \sum_{m}\left|P_{2, \text { norm }}-\bar{P}_{2, \text { norm }}\right| G_{m} / \sum_{m} G_{m}-\text { EIV }_{0}
$$

where $\bar{P}_{2, \text { norm }}$ is statistically given by

$$
\bar{P}_{2, \text { norm }} \approx \sqrt{2} \sum_{m} P_{2, m} G_{m} / \sqrt{\left[\sum_{m} P_{2, m}^{2} G_{m}\right]\left[\sum_{m} G_{m}\right]} .
$$

Equation (5a) is the key formulation to calculate the relationship between the normalized EIV and the dynamic void fraction. A constant term of $\mathrm{EIV}_{0}$ was introduced in the formulation to set the lowest limit of the void fraction that the sensor can detect. Although theory may predict very low void fractions corresponding to negligibly small values of EIVs, in practical situations, such EIVs cannot be measured because the SNR is not sufficiently high in such cases. The other reason why this term was introduced is that the calculated EIVs are heavily dependent on the frequency for a given void fraction and BSD, even when the void fraction is very low. The existence of a preset lowest detectable void fraction in the algorithm is important because the theory developed in the current study predicts the trend of the theoretical curves which relates the void fraction with the measured EIVs. Pragmatically, $\mathrm{EIV}_{0}$ is determined by the values which make the EIV zero at the minimum measurable values of the void fraction for a given frequency.

In order to investigate the contribution to the estimated EIV by a possible large gas pocket which is stationary in the liquid, consider a volume $V$ of liquid that consists of the measurement window of the Chi-beam sensor, in which there is gas space of volume $V_{\text {space}}$, and a volume $V^{\prime}$ of free-flowing bubbles. Averaging over volume $V$, there is a total void fraction of $\Gamma=\left(V_{\text {space }}+V^{\prime}\right) / V=\left(V_{\text {space }} / V\right)+\left(V^{\prime} / V\right)=\Gamma_{0}+\Gamma^{\prime}$, where $\Gamma_{0}=V_{\text {space }} / V$ is the "static" void fraction (the ratio to $V$ of the volume of gas that that does not move down the pipe) and $\Gamma^{\prime}=V^{\prime} / V$ is the dynamic void fraction. A bubble of a given size $R_{0 m}$ contributes $P_{2, m}$ to the Chi-beam signal that is a function of bubble size and quantity, i.e., $P_{2}\left(R_{0 m}, \Gamma\right)$. Consequently in the pipe system containing a total void fraction of $\Gamma=\Gamma_{0}+\Gamma^{\prime}$, Eq. (3) in this case becomes

$$
\mathrm{EIV} \approx \sum_{m}\left|P_{2, m}\left(\Gamma_{0}+\Gamma^{\prime}\right)-P_{2, m}\left(\Gamma_{0}\right)\right| G_{m} / \sum_{m} G_{m}
$$

where $P_{2, \text { nobub }}=P_{2, m}\left(\Gamma_{0}\right)$. Assuming that the dynamic void fraction is much smaller than the static one $\Gamma^{\prime} \ll \Gamma_{0}$, application of a Taylor expansion of the first order with respect to $\Gamma^{\prime}, P_{2, m}\left(\Gamma_{0}+\Gamma^{\prime}\right)$ expands as

$$
P_{2, m}\left(\Gamma_{0}+\Gamma^{\prime}\right) \approx P_{2, m}\left(\Gamma_{0}\right)+\left.\frac{\partial P_{2, m}(\Gamma)}{\partial \Gamma}\right|_{\Gamma=\Gamma_{0}} \Gamma^{\prime}
$$

Since this model requires the linear dependence of $P_{2, m}(\Gamma)$ on the void fraction $\Gamma$, then

$$
\left.\frac{\partial P_{2, m}(\Gamma)}{\partial \Gamma}\right|_{\Gamma=\Gamma_{0}} \Gamma^{\prime}=P_{2, m}\left(\Gamma^{\prime}\right)-P_{2, \text { nobub }}
$$

Therefore substitution of Eqs. (7) and (8) into Eq. (3) gives

$$
\mathrm{EIV} \approx \sum_{m}\left|P_{2, m}\left(\Gamma^{\prime}\right)-P_{2, \text { nobub }}\right| G_{m} / \sum_{m} G_{m}
$$

Equation (9) shows that the EIV obtained by the Chi-beam sensor is not influenced by the presence of gas in the space, and the EIV only measures the dynamic void fraction. There will of course be a limit to this, and when the volume of the gas space dominates in the sensing volume, the above assumptions will break down. This is physically realistic as one would not expect Chi-beam to be able to measure the bubble population in a thin film of liquid running along the bottom of an almost gas-filled pipe.

Although $P_{2}\left(R_{0 m}\right)$ is dependent on the insonification frequency, the pipe geometry and the material properties of the pipe and the liquid, the EIV is independent of these because of the normalization described in Eqs. (5a) and (5b). To examine the sensitivity of the estimate of dynamic void fraction derived from the EIV to the assumed shape of the bubble size distribution, Figs. 4(a) and 4(b) show simulations of the normalized signal levels, and Figs. 4(c) and 4(d) show their corresponding phases. This is done for the PMMA/ water tube described in Sec. II at $41 \mathrm{kHz}$ for the two bubble populations given by the $n_{b}\left(R_{0}\right)$ plotted in Figs. 4(e) and 4(f), respectively. Both have void fractions of $10^{-5}$. Figure 4(e) shows the shape of BSD commonly associated with bubble generation in highly turbulent environments, such as breaking ocean waves, ${ }^{36-41}$ and, for this example, the shape of the BSD is described by $n_{b}\left(R_{0}\right) \propto R_{0}^{-\alpha}$, where $\alpha=2.55$ and $R_{0}$ is sampled from $1 \mu \mathrm{m}$ to $1 \mathrm{~cm}$. Figure 4(f) plots a BSD that is a Boltzmann distribution with a peak bubble radius of $R_{p}=90 \mu \mathrm{m}$, with the shape of the BSD is described by $n_{b}\left(R_{0}\right) \propto R_{0} \exp \left(-R_{0} / R_{p}\right)$. Such a peaked population was previously measured using an optical method $^{10}$ in this pipe after gas injection through a vibrated needle. ${ }^{28,29}$ Note that the unit (bin size) of $n_{b}\left(R_{0}\right)$ is not critical in the calculation of Eqs. (3)-(5) since both numerator and denominator contain this term and the unit of $n_{b}\left(R_{0}\right)$ is cancelled out. However the shape of $n_{b}\left(R_{0}\right)$ is important since the contributions by the different sizes of bubbles, which is directly related with the shape of BSD, change the values of the EIV.

To show this, Figs. 4(a)-4(d) plot simulated time histories of the magnitude and phase of the Chi-beam signal by allowing the populations of bubbles [given by Figs. 4(e) and 4(f)] to pass through the measurement time window of Figs. 2(a) and 2(b). The time histories start with the smallest bubbles at $t=0 \mathrm{~s}$, and allow bubbles of increasing size to pass as time goes by, to end with the largest bubbles at $t=0.25 \mathrm{~s}$. It does not matter what order bubbles pass through the measurement time window, as shown by Eqs. (5a) and (5b). In this way, each time window corresponds to a particular size bin of bubbles with a specific number density. The upper horizontal axes of Figs. 4(e) and 4(f) show the temporal evolution of the BSD. 
The EIV is obtained by considering the surrounded areas by the magnitude of the Chi-beam signal [as denoted as dashed curves in Figs. 4(a) and 4(b)] and their averaged magnitude within the time window [as denoted as solid line in Figs. 4(a) and 4(b)]. The signal was designed to make the measured EIV robust with respect to the dependence on the bubble radii [Eqs. (5a) and (5b)]. To show this robustness, in Figs. 4(a) and 4(b) the contribution of each bubble to the EIV is modeled as proportional to $R_{0}^{l}$, where the above-mentioned quadratic dependence corresponds to $l=2$, while the value $l=1$ would be the case where a bubble's EIV is linearly dependent on bubble radius. For comparison, the value $l=0$ indicates the physically unrealistic condition that the bubble's contribution to the EIV is independent of its radius.

For each bubble as it passes through the sensor volume, the amplitude of the curve was obtained from Eq. (2) and the normalization from Eq. (5b). The normalized EIV is obtained by the time average of the area enclosed by the curves, $P_{2, \text { norm }}$ and $\bar{P}_{2, \text { norm }}$. Since for all curves the algorithm yields a value close to $\sqrt{2}$ for $\bar{P}_{2, \text { norm }}$, the value a sinusoid would produce, it is robust. Given that the curves in Fig. 4(a) all represent the same bubble population [that of Fig. 4(e)], then the robustness of the algorithm to the second order effects is clear: the normalized EIV, obtained by the time average of the area enclosed by the curves $P_{2, \text { norm }}$ and $\bar{P}_{2, \text { norm }}$, from Eq. (5a) should be similar for all curves in Fig. 4(a). The values do indeed agree to better than an order of magnitude, the yardstick for void fraction estimation industry: ${ }^{1}$ 0.0020 for $l=0,0.0129$ for $=1$, and 0.0108 for $l=2$. As expected, the output is similar for $l=1$ or 2 , but not for $l=0$. For the conditions of this experiment, $41 \mathrm{kHz}$ carrier wave and the BSD of Fig. 4(e), these are the EIV's which correspond to the dynamic void fraction of $\Gamma^{\prime}=3 \times 10^{-5}$. As a first estimate for calibration, this could be mapped onto the EIV $\sim 0.012$ obtained above for $l=1,2$. A similar degree of consistency is shown in Fig. 4(b), where for a $41 \mathrm{kHz}$ carrier wave and the BSD shown in Fig. 4(f), the dynamic void fraction $\Gamma^{\prime}=3 \times 10^{-5}$ gives EIV values: 0.0419 for $l=0,0.0283$ for $l=1$, and 0.0233 for $l=2$. Clearly, the choice of EIV to which $\Gamma^{\prime}=3 \times 10^{-5}$ corresponds depends on the shape of the BSD [ $\sim 0.012$ or $\sim 0.026$ for Figs. 4(c) and 4(d), respectively].

For the vertical pipes, the $l=2$ value was chosen in mapping the contribution of the bubbles to the EIV, which better accounted for the fact that larger bubbles rise faster than the smaller bubbles due to the buoyancy in the still water of that pipe. The two EIV estimates for the $l=2$ curves, 0.0108 in Fig. 4(a) and 0.0233 in Fig. 4(b), vary by $\sim 2.2$, typical of several simulations performed comparing bubble population shapes of the type shown in Figs. 4(e) and 4(f) for various void fractions. Without a priori knowledge of the shape of the BSD, the Chi-beam method is expected to give real time void fraction estimations of better than an order of magnitude, with a consistent (rather than varying) multiplicative discrepancy. This accuracy and sensitivity are adequate for some applications, but not others. The user must balance the achievable performance of the Chi-beam method against the affordability of, for example, using a greater bandwidth. ${ }^{10}$ In the ceramics industry, void fractions of less than $\sim 2 \times 10^{-5}$ are not detectable by a "compression test," the industry standard, ${ }^{1}$ which is neither real-time nor in-line. Resonator ${ }^{42}$ and impedance tube ${ }^{43}$ methods, although to date only deployed off-line, have the potential to be incorporated into pipelines and give higher accuracy than Chi-beam. However, they would not lend themselves to architectures like Chi-beam that could be rapidly moved from one section of the pipeline to another.

There have been very few attempts to measure bubbles in mercury, the goal of this series of papers. ${ }^{10,20,21,32}$ In the ceramics industry, the liquid is filled with many small particles which would create difficulties for many scattering techniques, but the bubble-induced reflective symmetry of the envelope perturbation allows Chi-beam to distinguish the particles from the bubbles. ${ }^{1}$ In the SNS TTF application, the liquid is again optically opaque, but the difficulty comes not from particles (which are not present in the mercury) but from reluctance of mercury to wet. Unlike Chi-beam, the resonator and impedance tube methods ${ }^{43-45}$ require good knowledge of the pipe and the boundary condition, which is not simple to attain when working with mercury. ${ }^{10}$ While Chi-beam simplifies this boundary condition problem, and provides an affordable, portable, real-time and in-line, nevertheless it retains are crucial drawback: the current use of $a$ priori knowledge of the shape of the BSD in Chi-beam is a clear limitation that could compromise ground-truthing. The implications of using Chi-beam will now be examined.

\section{RESULTS}

The open circles in Figs. 5(a) and 5(b) shows the averaged dynamic void fractions measured in the vertical PMMA pipe using the funnel collection technique. Error bars represent $+/-$ one standard deviation from the mean value of the ten repeated measurements. The dynamic void fraction was varied by changing the air supply pressure to the needle, and it is very important to note that this would almost certainly change the bubble size distribution. ${ }^{46}$ Since the shape of the bubble size distribution defines the mapping constant between the EIV and the dynamic void fraction, no prediction based on a single BSD shape will fit to the data. However, this scenario could well occur in SNS (although perhaps less so, because the sensing volume is much further downstream of the injection point), and so it is important to include such inconvenient effects.

Figures 5(a) and 5(b) also show the dynamic void fraction inferred from the measured EIV using various models for the shape of the BSD. The loci of solid dots plot out the calculations obtained from the theory introduced in Sec. III, and the solid curves are the fitted results by using zero phase filtering. Recall that Fig. 4(e) plots $n_{b}\left(R_{0}\right) \propto R_{0}^{-\alpha}$, where $\alpha=2.55$ and $R_{0}$ is sampled from $1 \mu \mathrm{m}$ to $1 \mathrm{~cm}$; and that Fig. 4(f) plots $n_{b}\left(R_{0}\right) \propto R_{0} \exp \left(-R_{0} / R_{p}\right)$, with the peak bubble radius chosen as $R_{p}=90 \mu \mathrm{m}$. The solid curves calculated in Fig. 5(a) also use $n_{b}\left(R_{0}\right) \propto R_{0}^{-\alpha}$ but here three different values of $\alpha$ are used: $\alpha=2.45,2.55$, and 2.65 as indicated in the picture. The solid curves in Fig. 5(b) use a Boltzmann distribution $n_{b}\left(R_{0}\right) \propto R_{0} \exp \left(-R_{0} / R_{p}\right)$ of the form shown in Fig. 4(f), although in Fig. 5(b) three different values of $R_{p}$ were used in the calculation of EIVs: $R_{p}=70,90$, and $110 \mu \mathrm{m}$. As the void fraction increases, the measured void fractions (the open 

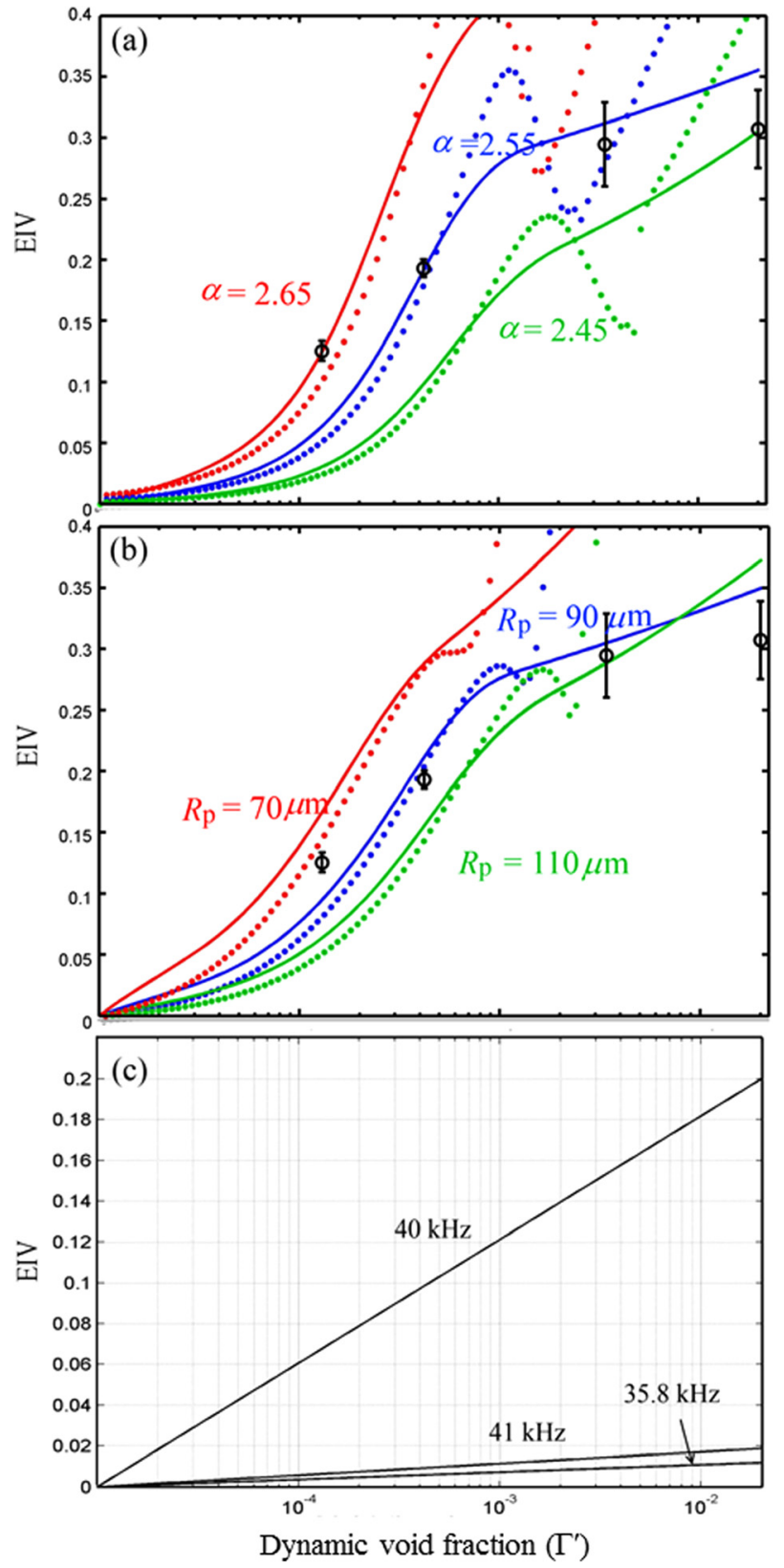

FIG. 5. (Color online) Panels (a) and (b) show theoretical calculations of EIVs vs dynamic void fraction in the PMMA/water tube at $41 \mathrm{kHz}$ for the shapes of $n_{b}\left(R_{0}\right)$ in Figs. 4(e) and 4(f), respectively. The index $l$ which shows the dependence of the counting rate of bubbles on the bubble radii is fixed as 2 throughout the calculation. The loci shown by solid dots demonstrates the results of the calculations for different choices of $\alpha$ or $R_{p}$. These are the parameters which characterize the bubble size distributions, as described in the caption of Fig. 4. The open circles are measurements at $41 \mathrm{kHz}$. The solid curves are simply fitted by the zero-phase filtering and not actual measurements. The average of the measured void fractions as a function of EIVs are denoted by open circles. The error bars represents $+/-$ one standard deviation from the mean value of the ten repeated measurements. Panel (c) shows the calibration data by the best fit solid line that would be used as the calibration curve for the ORNL SNS TTF test, were the wavenumber to be introduced into the formulation using the scheme described by Commander and Prosperetti (Ref. 11), the method described in this paper, at the three frequencies to be used at ORNL.

circles) tend to map to curves of different $\alpha$ in Fig. 5(a), and of different $R_{p}$ in Fig. 5(b), an expected feature given that the shape of the BSD is likely to change as greater void fractions are injected into the water column. With this in mind, when comparing between Figs. 5(a) and 5(b) the EIV is a surprisingly robust indicator of the dynamic void fraction, bearing in mind the drastically different assumed shapes of $n_{b}\left(R_{0}\right)$, given that physically realistic ranges of $\alpha$ and $R_{p}$ were chosen before the comparison was made. A word of caution is in order to emphasize the loss of accuracy at high dynamic void fractions. This is in keeping with all known acoustic techniques for measuring bubble populations, and here is in keeping with the inclusion in the algorithm of a theoretical model for acoustic propagation in bubbly water ${ }^{11}$ which was itself shown not to be accurate at higher dynamic void fractions.

Finally, Fig. 5(c) adapts the calibration curves that could be produced from Fig. 5(a), using the theory of Sec. III to convert them from relating to air bubbles in water, to apply to helium bubbles in the mercury of the SNS TTF pipeline. The calibration is shown by the best fit solid line that could be used if no better inversion algorithm is found before deploying the devices in an ORNL SNS TTF test at the three frequencies for which we have Chi-beam transducers. Where the smallest and largest bubbles in the calculation for air bubbles in water had radii of $1 \mu \mathrm{m}$ to $3 \mathrm{~mm}$, respectively, mapping the same bandwidth of resonance frequencies to helium bubbles in water require a bubble range from $0.25 \mu \mathrm{m}$ to $0.75 \mathrm{~mm}$ radius. In the absence of any measurements of the shape of the BSD, the process of bubble injection into turbulent flow suggests a bubble population some meters downstream of the form $n_{b}\left(R_{0}\right) \propto$ $R_{0}^{-\alpha}$ [Fig. 4(e)] with $\alpha=2.55$ chosen from the fit seen in Fig. 5 (a) for air bubbles in water. The calibration for the $40 \mathrm{kHz}$ transducer is very different from the calibrations for the frequencies on either side; the reason being the closeness of $40 \mathrm{kHz}$ to an $n=2$ resonance in the pipe (Fig. 6). Given the lighter damping of mercury in steel pipes compared to many other industrial pipelines, the existence of such resonances means that it would be sensible to perform measurements away from such resonances.

Independent measurement of the shape of the BSD would be highly beneficial when using Chi-beam, but in the absence of any such data from SNS TTF, the method of this paper can

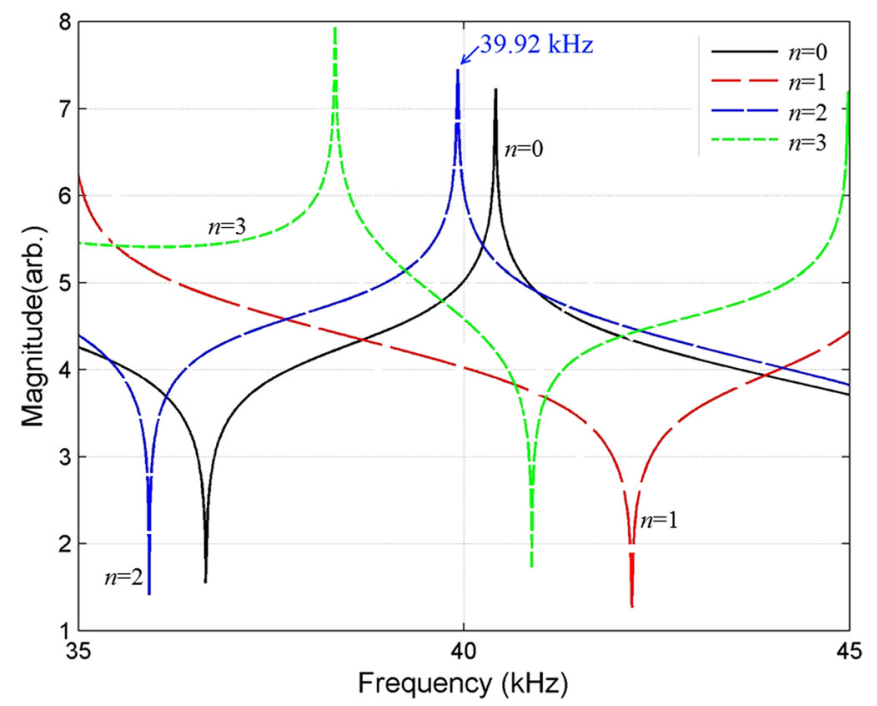

FIG. 6. (Color online) Simulation of the predicted response function of the steel pipe to circumferential modes that are coupled between the pipe wall and its content (mercury), when the pipe contains a helium bubble population resembling that shown in Fig. 4(e), for $n_{b}\left(R_{0}\right) \propto R_{0}^{-\alpha}$ with $\alpha=2.55$. 
only be used via an estimate. As stated above, dynamic void fractions of greater than $\sim 10^{-3}$ should be critically examined because of the increasing inaccuracy expected at high void fraction in both estimation method and the theoretical modeling. If there were access to an appropriate independent measure of void fraction, then calibration curves such as Fig. 5(c) could be adjusted at the high void fraction end with empirical measurements. However, the accuracy of the estimation will be limited in most circumstances since the EIV will tend to plateau at high void fraction [as in Figs. 5(a) and 5(b)], so that large changes in the dynamic void fraction produce only small changes in the EIV. Consequently, at high void fractions, EIV becomes an insensitive measure of void fraction.

\section{CONCLUSIONS}

In terms of the objectives stated at the end of Sec. I, (i) and (ii) have been achieved. A suitable detection signal was found, which relied on perturbation of the carrier wave, chosen such that noise cannot be mistaken for the presence of bubbles (Fig. 2). Objective (iii) was to explore whether this signal could be suitably inverted to determine the dynamic void fraction, and most importantly to answer the question that should be asked of all such inversion, "what a priori information is required to achieve an acceptable level of reliability?" This question is crucial since, if the required a priori information is not available from the industrial system for which it is designed, or if it requires input from data that is later used for ground-truthing, the method is unacceptable.

The concept of "dynamic void fraction" is critical for the purpose of mass transfer in bioreactors, product quality in the manufacture of food products, and the absorption of shock waves in bulk liquid at ORNL. Such absorption requires that the bubbles (i) are present in the bulk of the liquid rather than trapped on the walls and (ii) change volume under the pressure wave so that the acoustic energy is converted, ultimately, to heat through viscous losses at the bubble wall, and adiabatic heating of the compressed bubble gas. Both conditions require that the bubble not be too large: buoyancy forces are stronger on larger bubbles, and in order to undergo significant volume change the bubble response time, which is more rapid for smaller bubbles, ${ }^{27}$ must not be longer than the timescales over which the rarefactions and compressions in the pressure pulse. The "dynamic void fraction" refers to the proportion of the bubbly liquid which is in the form of bubble gas moving with the flow: large stationary pockets of gas (trapped, for example, at junctions in pipes, or occupying the upper half of a horizontal pipe which contains liquid only in its lower half), do not contribute to the gas volume in the numerator of the "dynamic void fraction."

This paper records the development of a sensor to measure the dynamic void fraction in real time. The work constituted the half-way point in a two-year project, the first year having been aimed at a broadband sensor that would provide unambiguous results. ${ }^{10,20,21}$ Sponsor budget constraints half way through the contract required a completely different approach, and although the eventual sensor overcame many of the difficulties in the specification (greatly reduced cost, and was later shown to be able to transmit into mercury without chemically affecting it) and came with valuable additional benefits not in the original specification (real time operation, ability to operate through the pipe walls and be easily relocated, ability to operate without a bubble-free datum reading), nevertheless one difficulty remained, and that was the need to input the algorithm with the shape (but not the absolute level of) the bubble size distribution present. If no independent measure of this shape is possible, as was the case at ORNL SNS TTF at the time, then an estimate must be made of which of the available options is most suitable. It is always important to emphasize what a priori knowledge is required for the algorithm-here is it is shape of the bubble size spectrum, but not the absolute number of bubbles, and hence not the dynamic void fraction that is the output of this sensor. Ideally SNS TTF would have independent (e.g., optical) measures of that spectral shape. The authors and their colleagues have tried two optical techniques: (i) analyzing the bubble populations that rise through mercury to become visible against windows in the pipeline and (ii) inserting optical fibers into the mercury and monitoring the scatter at the remote end. While both techniques were successful at detecting bubbles, they could not be relied upon to provide statistically accurate BSDs. Dual electrode techniques (one electrode to monitor for cavitation erosion, the other for bubble presence $)^{47}$ suffered from similar drawbacks, and others associated with the conductivity of mercury and the need not to contaminate it.

It cannot be said that the search for a reliable algorithm to invert the Chi-beam output into absolute dynamic void fractions is complete: most industrial applications would require an algorithm that is more robust in terms of details of the assumed conditions. With the current method of incorporating the wavenumber into the formulation using the approach of Commander and Prosperetti, ${ }^{11}$ the Chi-beam sensor will give uncertain values of the void fractions if the shape of the bubble size distribution is not known. As an illustration, if so little is known about the distribution that it could conform to any of the curves in Fig. 5(a) or 5(b) (but with the limits on $\alpha$ and $R_{p}$ given by the range used to plot those figures), then the maximum dynamic void fraction that a given EIV could represent could be 5-10 times greater than the minimum void fraction (provided the maximum void fraction is less than around $10^{-3}$ ).

If this requirement for a priori knowledge of the shape of the BSD can be overcome, as a practical tool Chi-beam compares favorably with the option of using the standard free field broadband inversion technique, which for pipe rigs such as SNS TTF was demonstrated ${ }^{10}$ to produce errors as great as $+/-$ $1000 \%$. Chi-beam provides a useful real-time low cost tool for industries that value an estimate of void fraction that is accurate to better than an order of magnitude, and faithfully reports on relative changes in the dynamic void fraction. However, for a reliable estimate of the absolute void fraction that does not require a priori knowledge of the bubble size distribution, a method based on the reduction in sound speed seen when all bubbles are smaller than resonance $e^{15,48,49}$ might be preferable.

\section{ACKNOWLEDGMENTS}

This work is co-funded by Oak Ridge National Laboratory, Tennessee, US, and (ii) the UK Science \& Tech. 
Res. Council (Principle Investigator: T.G.L.). The authors are grateful to B. Riemer, M. Wendel. R. Sangrey, C. Densham, O. Caretta, and T. Davenne.

${ }^{1}$ G. T. Yim and T. G. Leighton, "Real-time on-line monitoring of ceramic 'slip' in pottery pipe-lines using ultrasound," Ultrasonics 50, 60-67 (2010). ${ }^{2}$ G. M. Campbell and E. Mougeot, "Creation and characterisation of aerated food products," Trends Food Sci. Technol. 10, 283-296 (1999).

${ }^{3}$ W. Punurai, J. Jarzynski, J. Qu, K. E. Kurtis, and L. J. Jacobs, "Characterization of entrained air voids in cement paste with scattered ultrasound," NDT \& E Int. 39, 514-524 (2006).

${ }^{4}$ T. G. Leighton and P. R. White, "Quantification of undersea gas leaks from carbon capture and storage facilities, from pipelines and from methane seeps, by their acoustic emissions," Proc. R. Soc. London, Ser. A 468, 485-510 (2012).

${ }^{5}$ E. P. Stride and C. C. Coussios, "Cavitation and contrast: The use of bubbles in ultrasound imaging and therapy," Proc. Inst. Mech. Eng., Part H 224, 171-191 (2010).

${ }^{6}$ A. O. Maksimov and T. G. Leighton, "Pattern formation on the surface of a bubble driven by an acoustic field," Proc. R. Soc. London, Ser. A 468, 57-75 (2012).

${ }^{7}$ D. G. Offin, P. R. Birkin, and T. G. Leighton, "Electrodeposition of copper in the presence of an acoustically excited gas bubble," Electrochem. Commun. 9, 1062-1068 (2007).

${ }^{8} \mathrm{D}$. Felde, B. Riemer, and M. Wendel, "Development of a gas layer to mitigate cavitation damage in liquid mercury spallation targets," J. Nucl. Mater. 377, 155-161 (2008).

${ }^{9}$ P. V. Chitnis, N. J. Manzi, R. O. Cleveland, R. A. Roy, and R. G. Holt, "Mitigation of damage to solid surfaces from the collapse of cavitation bubble clouds," J. Fluids Eng. 132, 051303 (2010).

${ }^{10}$ T. G. Leighton, K. Baik, and J. Jiang, "The use of acoustic inversion to estimate the bubble size distribution in pipelines," Proc. R. Soc. London, Ser. A 468, 2461-2484 (2012).

${ }^{11}$ K. W. Commander and A. Prosperetti, "Linear pressure waves in bubbly liquids: Comparison between theory and experiments," J. Acoust. Soc. Am. 85, 732-746 (1989).

${ }^{12} \mathrm{~K}$. W. Commander and R. J. McDonald, "Finite-element solution of the inverse problem in bubble swarm acoustics," J. Acoust. Soc. Am. 89, 592-597 (1991).

${ }^{13}$ R. Duraiswami, S. Prabhukumar, and G. L. Chahine, "Bubble counting using an inverse acoustic scattering method," J. Acoust. Soc. Am. 104, 2699-2717 (1998).

${ }^{14}$ T. G. Leighton, S. D. Meers, and P. R. White, "Propagation through nonlinear time dependent bubble clouds, and the estimation of bubble populations from measured acoustic characteristics," Proc. R. Soc. London, Ser. A 460, 2521-2550 (2004).

${ }^{15}$ T. G. Leighton and G. B. N. Robb, "Preliminary mapping of void fractions and sound speeds in gassy marine sediments from subbottom profiles," J. Acoust. Soc. Am. 124, EL313-EL320 (2008).

${ }^{16}$ J. W. L. Clarke and T. G. Leighton, "A method for estimating timedependent acoustic cross-sections of bubbles and bubble clouds prior to the steady state," J. Acoust. Soc. Am. 107, 1922-1929 (2000).

${ }^{17}$ J. Cui, M. F. Hamilton, P. S. Wilson, and E. A. Zabolotskaya, "Bubble pulsations between parallel plates," J. Acoust. Soc. Am. 119(4), 2067-2072 (2006).

${ }^{18} \mathrm{~T}$. G. Leighton, "The inertial terms in equations of motion for bubbles in tubular vessels or between plates," J. Acoust. Soc. Am. 130, 3333-3338 (2011).

${ }^{19}$ T. G. Leighton, D. G. Ramble, and A. D. Phelps, "The detection of tethered and rising bubbles using multiple acoustic techniques," J. Acoust. Soc. Am. 101(5), 2626-2635 (1997).

${ }^{20}$ K. Baik, J. Jiang, and T. G. Leighton, "Acoustic attenuation, phase and group velocities in liquid-filled pipes: Theory, experiment, and examples of water and mercury," J. Acoust. Soc. Am. 128, 2610-2624 (2010).

${ }^{21}$ J. Jiang, K. Baik, and T. G. Leighton, "Acoustic attenuation, phase and group velocities in liquid-filled pipes II. Simulation for spallation neutron sources and planetary exploration," J. Acoust. Soc. Am. 130, 695-706 (2011).

${ }^{22}$ T. G. Leighton, P. R. White, and M. A. Marsden, "Applications of onedimensional bubbles to lithotripsy, and to diver response to low frequency sound," Acta Acust. 3(6), 517-529 (1995).

${ }^{23}$ T. G. Leighton, W. L. Ho, and R. Flaxman, "Sonoluminescence from the unstable collapse of a conical bubble," Ultrasonics 35, 399-405 (1997).

${ }^{24}$ T. G. Leighton, B. T. Cox, and A. D. Phelps, "The Rayleigh-like collapse of a conical bubble," J. Acoust. Soc. Am. 107(1), 130-142 (2000).
${ }^{25}$ T. G. Leighton, D. G. Ramble, A. D. Phelps, C. L. Morfey, and P. P. Harris, "Acoustic detection of gas bubbles in a pipe," Acust. Acta Acust. 84, 801-814 (1998).

${ }^{26}$ T. G. Leighton, P. R. White, C. L. Morfey, J. W. L. Clarke, G. J. Heald, H. A. Dumbrell, and K. R. Holland, "The effect of reverberation on the damping of bubbles," J. Acoust. Soc. Am. 112, 1366-1376 (2002).

${ }^{27}$ T. G. Leighton, "What is ultrasound?," Prog. Biophys. Mol. Biol. 93, 3-83 (2007).

${ }^{28}$ T. G. Leighton, J. Jiang, and K. Baik, "A TV demonstration of sound absorption connecting the space shuttle to submarines," Acoust. Bull. 36, 35-40 (2011).

${ }^{29}$ T. G. Leighton, J. Jiang, and K. Baik, "Demonstration comparing sound wave attenuation inside pipes containing bubbly water and water droplet fog," J. Acoust. Soc. Am. 131(3), 2413-2421 (2012).

${ }^{30}$ R. Clift, J. R. Grace, and M. E. Weber, Bubbles, Drops, and Particles (Academic Press, New York, 1978), pp. 171-173.

${ }^{31}$ T. G. Leighton, The Acoustic Bubble (Academic Press, London, 1994), pp. 146-148.

${ }^{32}$ K. Baik, J. Jiang, and T. G. Leighton, "Acoustic attenuation, phase and group velocities in liquid-filled pipes III: Non-axisymmetric propagation and circumferential modes in lossless conditions," J. Acoust. Soc. Am. 133(2), 1225-1236 (2013).

${ }^{33}$ T. G. Leighton, K. Baik, and J. Jiang, "Theoretical background for a potential real time quantification of gas bubbles in pipelines," ISVR Technical Report No. TR 335, The Institute of Sound and Vibration Research, University of Southampton, Southampton, UK, 2014.

${ }^{34}$ M. A. Ainslie and T. G. Leighton, "Review of theory for scattering and extinction cross-sections, damping factors and resonance frequencies of spherical gas bubbles," J. Acoust. Soc. Am. 130(5), 3184-3208 (2011).

${ }^{35}$ M. A. Ainslie and T. G. Leighton, "Near resonant bubble acoustic crosssection corrections, including examples from oceanography, volcanology, and biomedical ultrasound," J. Acoust. Soc. Am. 126(5), 2163-2175 (2009).

${ }^{36}$ T. G. Leighton, D. C. Finfer, P. R. White, G.-H. Chua, and J. K. Dix, "Clutter suppression and classification using Twin Inverted Pulse Sonar (TWIPS)," Proc. R. Soc. London, Ser. A 466, 3453-3478 (2010).

${ }^{37}$ N. D. Breitz and H. Medwin, "Instrumentation for in situ acoustical measurements of bubble spectra under breaking waves," J. Acoust. Soc. Am. 86, 739-743 (1989).

${ }^{38}$ D. M. Farmer and S. Vagle, "Waveguide propagation of ambient sound in the ocean-surface bubble layer," J. Acoust. Soc. Am. 86, 1897-1908 (1989).

${ }^{39}$ G. B. Deane and M. D. Stokes, "Air entrainment processes and bubble size distributions in the surf zone," J. Phys. Oceanogr. 29, 1393-1403 (1999).

${ }^{40}$ A. D. Phelps and T. G. Leighton, "Oceanic bubble population measurements using a buoy-deployed combination frequency technique," IEEE J. Oceanic Eng. 23, 400-410 (1998).

${ }^{41}$ C. Garrett, M. Li, and D. Farmer, "The connection between bubble size spectra and energy dissipation rates in the upper ocean," J. Phys. Oceanogr. 30, 2163-2171 (2000).

${ }^{42}$ D. M. Farmer, S. Vagle, and D. Booth, "Reverberation effects in acoustical resonators used for bubble measurements," J. Acoust. Soc. Am. 118, 2954-2960 (2005).

${ }^{43}$ P. S. Wilson, R. A. Roy, and W. M. Carey, "Phase speed and attenuation in bubbly liquids inferred from impedance measurements near the individual bubble resonance frequency," J. Acoust. Soc. Am. 117, 1895-1910 (2005).

${ }^{44}$ C. Ormonde, P. Chitnis, R. O. Cleveland, R. G. Holt, and R. A. Roy, "A vertical acoustic waveguide for two-phase mercury-helium flow void fraction determination," IMECE2008, abstract No. 66791 (American Society of Mechanical Engineers, New York, 2008), p. 196.

${ }^{45}$ R. A. Roy, C. E. Ormonde, P. V. Chitnis, R. O. Cleveland, and R. G. Holt, "An acoustic resonator for determining the void fraction of bubbly mercury flows," J. Acoust. Soc. Am. 123, 3559 (2008).

${ }^{46}$ T. G. Leighton, K. J. Fagan, and J. E. Field, "Acoustic and photographic studies of injected bubbles," Eur. J. Phys. 12, 77-85 (1991).

${ }^{47}$ P. R. Birkin, D. G. Offin, P. F. Joseph, and T. G. Leighton, "Cavitation, shock waves and the invasive nature of sonoelectrochemistry," J. Phys. Chem. B 109, 16997-17005 (2005).

${ }^{48}$ P. S. Wilson, A. H. Reed, W. T. Wood, and R. A. Roy, "The lowfrequency sound speed of fluid-like gas-bearing sediments," J. Acoust. Soc. Am. 123, EL99-EL104 (2008).

${ }^{49}$ P. R. Birkin, T. G. Leighton, J. F. Power, M. D. Simpson, A. M. L. Vincotte, and P. Joseph, "Experimental and theoretical characterisation of sonochemical cells. Part 1. Cylindrical reactors and their use to calculate the speed of sound in aqueous solutions," J. Phys. Chem. A 107, 306-320 (2003). 